\title{
Comportamiento fenológico de Wercklea insignis (Malvaceae) en un Bosque Pluvial Premontano, San Ramón, Costa Rica
}

\author{
Phenological behavior of Wercklea insignis (Malvaceae) in a Premontane Rain Forest, San Ramón, \\ Costa Rica
}

\section{Liz Brenes Cambronero ${ }^{1}$}

Fecha de recepción: 18-12-2019

Fecha de aceptación: 28-09-2020

\begin{abstract}
Resumen
Se estudió la fenología de Wercklea insignis, árbol con potencial importante por sus flores conspicuas, que crece a orilla de los ríos, en la Reserva Biológica Alberto Manuel Brenes, Alajuela, Costa Rica. La evaluación se realizó de junio de 2014 hasta octubre de 2015. Se monitorearon 10 individuos con producción de flores, con un diámetro a la altura del pecho de $7 \mathrm{~cm}$ a $11.9 \mathrm{~cm}$, mientras que la altura de los árboles osciló entre $8 \mathrm{~m}$ y $15 \mathrm{~m}$. Cada espécimen de $W$. insignis se enumeró con marcas plásticas, luego se georreferenciaron cada uno de ellos. Las fenofases de floración (subanual), brotes foliares, caída de follaje fueron continuas, sin perder las hojas completamente. En tanto que la fructificación estuvo ausente solo en octubre 2014 y junio 2015. Existió asincronía entre individuos en el comportamiento de las etapas fenológicas. La caída de follaje ( $\mathrm{n}=10, \mathrm{r}=0.35, \mathrm{p}<0.05)$ y el nacimiento de flores $(n=10, r=0.46, p<0.05)$ se correlacionaron con la precipitación; mientras que el brote de frutos $(n=10, r=-0.51, p<0.05)$ tuvo un vínculo inversamente proporcional con este fenómeno.
\end{abstract}

Palabras claves: Fenología, fenofases, Reserva Biológica Alberto Manuel Brenes, floración subannual.

\begin{abstract}
I studied the phenology of $W$. insignis ( $\mathrm{n}=10$ ), tree with great ornamental potential with conspicuous flowers, which grows on the San Lorencito riverbed, in the Alberto Manuel Brenes Biological Reserve, Alajuela, Costa Rica, June 2014 until October 2015. Of the selected sample of six individuals with flower production, the diameter at breast height ranged from $7 \mathrm{~cm}$ to $11.9 \mathrm{~cm}$, while the highest trees measured $15 \mathrm{~m}$. The identification of the individuals was done with plastic marks enumerated, after this they were georeferenced. In relation the flowering phenotypes (subanual), foliar sprouts, fall foliage were continuous, without having a total loss of the leaves. While the fructification was absent in October 2014 and June 2015. There was asynchrony between individuals in the behavior of the phenological stages. The fall of foliage $(n=10, r=0.35, p<0.05)$ and flowering $(n=10, r=$ $0.46, \mathrm{p}<0.05)$ correlated with precipitation while fruiting $(\mathrm{n}=10), \mathrm{r}=-0.51, \mathrm{p}<0.05)$ had an inversely proportional correlation with this phenomenon.
\end{abstract}

Key words: Phenology, phenofases, Alberto Manuel Biologic Reserve, subannual flowering.

\footnotetext{
1 Docente investigadora en la Universidad de Costa Rica, Sede de Occidente, Sección de Biología, Costa Rica. Programa de Investigaciones en Gestión Sostenible de los Recursos Naturales. Correo electrónico: 1lmbrenes@hotmail.com
} 


\section{Introducción}

El género Wercklea pertenece a la familia Malvaceae y posee 13 especies (Standley, 1937), las cuales crecen en hábitats relativamente húmedos como orillas de ríos, bosques de lluvia o de niebla. La distribución geográfica del género alcanza por el norte a Costa Rica y las Antillas, mientras que por el sur llega a Colombia y Ecuador; se conocen también ejemplares de Bolivia. El mayor número de especies se encuentra en Centroamérica (Standley y Pittier, 1916).

Wercklea es un género endémico compuesto de dos especies, una de ellas, Wercklea insignis (Malvaceae) (Standley y Pittier, 1916), ya ha sido plantada en algunos jardines de distintos sitios de Costa Rica. W. insignis, conocida como clavelón de montaña o burío, tiene hojas alternas las cuales miden $20 \mathrm{~cm}$ de largo, de forma acorazonada. Su flor es conspicua con tonalidades de rosado a lavanda (Standley, 1937), por lo que representa potencial importante como especie ornamental en diferentes espacios, ya sea parques, aceras y otros lugares públicos o privados.

Esta especie se encuentra en la categoría vulnerable en su distribución natural, la cual es Costa Rica y el oeste de Panamá (Standley, 1937). Dentro de las escasas investigaciones sobre este árbol se puede citar la realizada por Sáenz y Nassar (1965), quienes determinaron mediante un análisis cualitativo que $W$. insignis no contiene alcaloides en hojas o tallos.

Originalmente $W$. insignis fue denominada como Hibiscus wercklei. El género Hibiscus tiene usos económicos como fibra, vinos, siropes (Mahadevan, 2009), cuidados del cabello, té de hierbas, jalea (Cheng, 2001), papel (Mohta, 2000) y medicinal (Abdallah, 2011) (Cheng, 2001) (Odigie y Adigun, 2003). Por lo cual se considera que $W$. insignis puede tener múltiples usos. Fryxell (1981) señaló que muchas especies de Wercklea tienen una o más características únicas y esenciales para este género, por ejemplo, $W$. insignis desarrolla una madera liviana excepcional comparables con el corcho. Además, de la corteza se extraen fibras usadas como cuerdas para amarrar (Pérez y Condit, 2017).
Dentro de los estudios que deben desarrollarse para conocer la autoecología de una especie, está la fenología (Brenes y Di Stéfano, 2001), la cual según Pérez, Villalba y Almanza (2011), tiene un rol importante en el desarrollo de la humanidad. Con base en esta información, ha sido posible predecir y planificar los recursos para su sostenimiento. Los pueblos recolectores y cazadores han elaborado calendarios para el acopio de frutos silvestres y para la caza, sistema que les permite comprender los ciclos biológicos de su ambiente (LaRotta, 1987).

También, se considera vital la comprensión de estos fenómenos biológicos, puesto que aportan conocimiento sobre las relaciones entre las plantas y los animales de una comunidad biótica y sus vecinas (Fournier y Charpantier, 1975). Además, entender los patrones fenológicos ayuda a la conservación de los recursos genéticos y al manejo forestal, porque describe los momentos de desarrollo vegetativo, temporadas de reproducción, cosecha y dispersión de semillas (Mooney et al., 1980; Huxley, 1983; Rondón, 1992; Gómez, 2010). En este caso, coopera con el entendimiento de $W$. insignis, la cual es, además, una especie endémica.

Un primer estudio de la fenología de cualquier especie puede servir como base para posteriores investigaciones y así comprender cómo los individuos se adaptan al cambio climático. De acuerdo con Estrada et al. (2015), este fenómeno significa, en términos ambientales, que muchas de las poblaciones de plantas, en su intento por sobrevivir a nuevas condiciones, van a tener cambios para poder aclimatarse.

Así, el objetivo del presente trabajo consistió en investigar las fenofases de Wercklea insignis (Malvaceae) en un Bosque Pluvial Premontano en la Reserva Biológica Alberto Manuel Brenes. Además, se estimó el grado de correlación entre las fenoetapas de caída de follaje, presencia de brotes foliares, nacimiento de flores y surgir de frutos con la temperatura, humedad relativa y precipitación. 


\section{Materiales y métodos}

Esta investigación se llevó a cabo entre junio de 2014 hasta octubre de 2015 en la Reserva Biológica Alberto Manuel Brenes, San Ramón, Alajuela, Costa Rica, ubicada en la parte sur de la Cordillera de Tilarán (coordenadas CRTMo5 0439494E y 1131058N). La precipitación promedio es de $3500 \mathrm{~mm}$, su temperatura promedio es de $21^{\circ} \mathrm{C}$ y corresponde a la zona de vida Bosque Pluvial Premontano (Brenes, 1999).

Se estudió una muestra de 10 árboles de $W$. insignis (Malvaceae) (L. Brenes Cambronero, 2017 (USJ)), seleccionados por orden de aparición y con un diámetro, a la altura del pecho, igual o superior a $7 \mathrm{~cm}$, los cuales, además, poseyeran flores. Para identificar a $W$. insignis, se conoce que mide de $5 \mathrm{~m}$ a $15 \mathrm{~m}$ de altura, se encuentra por lo general a orilla de ríos en bosques húmedos entre 1000 msnm y 3000 msnm (Pérez y Condit, 2017), aunque en este estudio se presenció a $850 \mathrm{msnm}$. Sus hojas son alternas, de $20 \mathrm{~cm}$ de largo, acorazonadas y poseen flores conspicuas de color rosado a lavanda (Standley, 1937) (Figura 1).
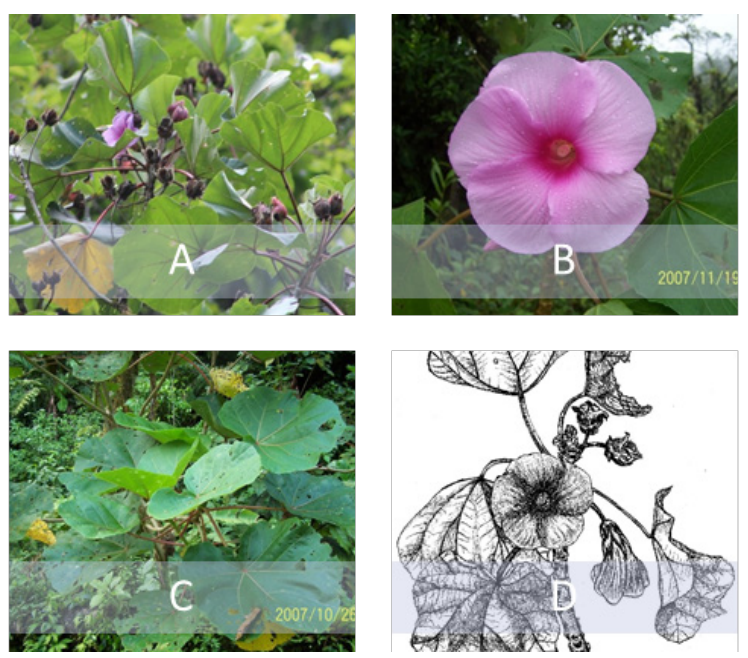

Figura 1. Flores y frutos (A), floración (B), follaje (C). Ilustración de Juan Gabriel Parra López (D) de $W$. insignis $(\mathrm{n}=10)$. Imágenes tomadas de junio 2014 a octubre 2015. Sendero La Catarata, Reserva Biológica Alberto Manuel Brenes, Costa Rica.
Estos árboles estaban localizados en promedio a 320 metros de la Estación Biológica Rodolfo Ortiz Vargas, a orillas del río San Lorencito y en el sendero La Catarata. La distancia existente, en línea recta, entre el primer y último árbol fue de $250 \mathrm{~m}$ (Figura 2).

Ubicación de los individuos de Wercklea insignis en la ReBAMB

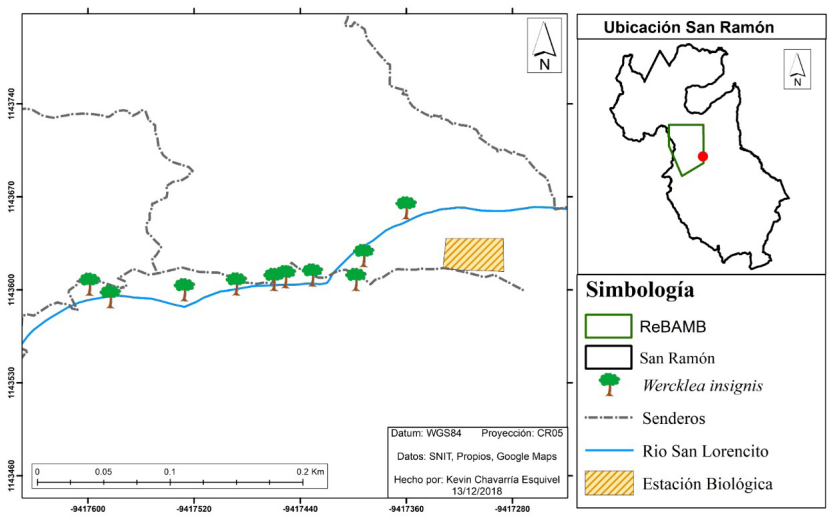

Figura 2. Ubicación de árboles $W$. insignis $(\mathrm{n}=10)$, sendero La Catarata, Reserva Biológica Alberto Manuel Brenes, Costa Rica.

La muestra seleccionada fue de 10 individuos de acuerdo con Fournier y Charpantier (1975), quienes afirman que para el estudio fenológico de la vegetación tropical es recomendable el empleo de un corpus de 10 ejemplares por especie, elegidos por orden de aparición en el bosque. El grupo resultante tenía un diámetro, a la altura del pecho, de $7 \mathrm{~cm}$ a $11.9 \mathrm{~cm}$, mientras que la altura de los árboles osciló entre $8 \mathrm{~m}$ a $15 \mathrm{~m}$ (Cuadro 1). 
Cuadro 1. Altura y diámetro de los ejes observados de $W$. insignis $(\mathrm{n}=10)$, ReBAMB, Costa Rica. Junio 2014 a octubre 2015.

\begin{tabular}{|l|l|l|}
\hline No. Individuo & Altura $X(\mathrm{~m})$ & Diámetro X $(\mathrm{cm})$ \\
\hline 1 & 15 & 10 \\
\hline 2 & 8 & 7.1 \\
\hline 3 & 10 & 8 \\
\hline 4 & 15 & 11.9 \\
\hline 5 & 15 & 9.6 \\
\hline 6 & 12 & 7 \\
\hline 7 & 8 & 8.5 \\
\hline 8 & 13 & 10 \\
\hline 9 & 10 & 8 \\
\hline 10 & 11 & 10 \\
\hline Media & 11.8 & 9 \\
\hline
\end{tabular}

Cada individuo de $W$. insignis se enumeró con marcas plásticas y posteriormente se georreferenció (Figura 2). A cada árbol se le evaluaron dos fases fenológicas vegetativas: caída de follaje (cf) y aparición de brotes foliares (br); además de dos reproductivas: nacimiento de flores (fl) y el emerger de frutos (fr); ambas con el uso de binoculares desde diferentes perspectivas y ángulos. La toma de datos de este comportamiento se hizo una vez al mes y se utilizó una escala de o a 4 : donde o equivale a la ausencia de la etapa fenológica, 1 corresponde desde el $1 \%$ hasta el 25\%, 2 desde $26 \%$ hasta $50 \%$, 3 desde $51 \%$ a $75 \%$ y 4 de $76 \%$ al $100 \%$ en cuanto a presencia de la fenoetapa (Fournier, 1974).

Se estimaron correlaciones lineales con base en el promedio de cifras, de un periodo de diez años, según la estación meteorológica de la ReBAMB. La humedad relativa, la lluvia y la temperatura se vincularon con caída de follaje, surgimiento de brotes foliares, desarrollo floral y fructificación.

\section{Resultados}

Durante el estudio, $W$. insignis siempre presentó caída de follaje, sin embargo, esta especie nunca tuvo una pérdida total de hojas. El pico máximo de este fenómeno en $W$. insignis fue en noviembre de 2014 , en este momento, descendió el nacimiento de flores y ascendió la fructificación (Figura 3).

También se registraron durante la investigación los brotes foliares, cuyo valor mínimo promedio fue de 0.4. Después se presentaron dos momentos constantes (agosto 2014 a diciembre 2014 y agosto 2015 a octubre 2015) en donde la cifra fenológica fue de 1. Es importante resaltar que esta fenofase osciló apenas entre 0.4 y 1.7. Lo anterior refleja que los árboles tuvieron pocos brotes foliares a la vez, los cuales responden a la caída de follaje y al crecimiento vegetativo, al parecer constantes.

Ahora bien, la floración de esta especie de árbol siempre se presentó, con el valor mínimo de o.6 y el máximo de 3 en septiembre 2014. En este momento, la muestra estudiada no tenía frutos. Por su parte, la fructificación estuvo ausente apenas en octubre 2014 y junio 2015, cuyo valor promedio máximo fue de 2.6 en junio 2014. Esta especie se encuentra en el margen o cauce de los ríos (Figura 1), los cuales pueden facilitar la propagación de frutos y semillas.

Por tanto, este árbol demostró un comportamiento dinámico durante el tiempo de estudio, aunque ninguna de las fenoetapas investigadas alcanzó cifras superiores a 3. Cabe destacar que esta especie parece tener periodos mensuales en donde manifiesta la actividad de las cuatro fases fenológicas como por ejemplo de diciembre 2014 a junio 2015, con valores relativamente bajos en un ámbito de 2.1 a 1.1 (Figura 3).

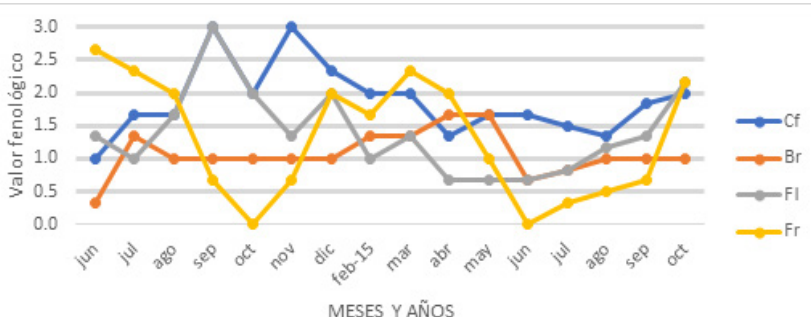

Figura 3. Dendrofenograma de W.insignis $(\mathrm{n}=10)$ junio de 2014 a octubre 2015. Reserva Biológica Alberto Manuel Brenes, Costa Rica. (cf: caída de follaje, br: brotes foliares, fl: floración, fr: fructificación). 
En general, el comportamiento fenológico que compuso la muestra fue diferente entre individuos. Como ejemplo, se observa en los ejemplares 1, 3 y 5 la caída de follaje durante todo el periodo de observación, excepto en junio 2014, en donde el espécimen 5 no presentó esta fenofase. También llama la atención el mes de septiembre, el cual alcanzó el valor máximo en el promedio, además de individualmente en las especies 1 y 5 . En la media calculada de la muestra, la caída de follaje osciló entre los valores de 1 y 3 . (Figuras 3 y 4 ).

Individuo 1

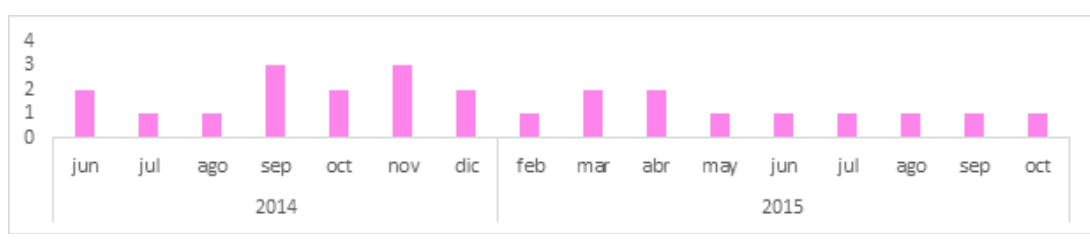

Individuo 3

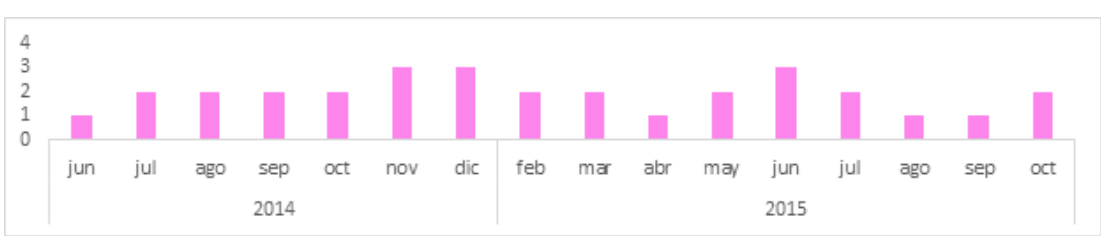

Individuo 5

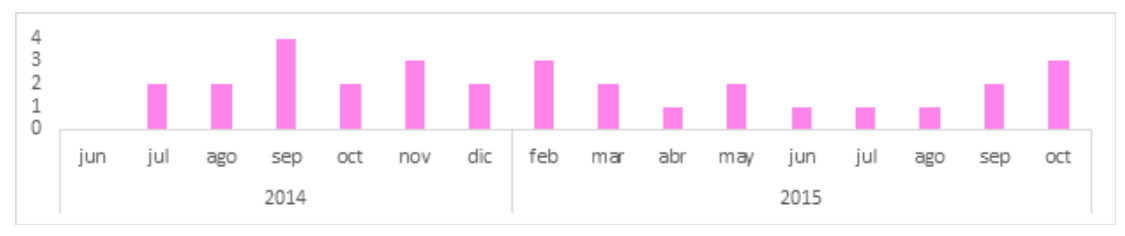

Promedio

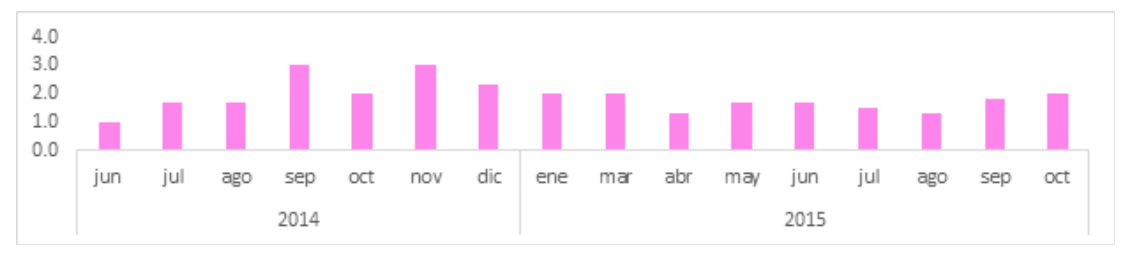

Figura 4. Promedio total (T) $(n=10)$ de caída de follaje en tres de los 10 individuos estudiados y tomados al $\operatorname{azar}(1,3,5$ de $W$. insignis). 
$W$. insignis produjo brotes foliares durante el periodo de estudio y mostró una menor producción en junio de ambos años, cuyos valores fueron 0.3 en 2014 y 0.7 para 2015. Esta especie presentó este fenómeno de manera constante con un valor de 1 para el periodo de agosto a diciembre 2014 y de agosto a octubre 2015.
Los valores registrados de brotación tuvieron un ámbito de 0.3 a 1.7 en la media. No obstante, en algunos individuos fue de o a 2. Los meses con mayor presencia de brotes foliares fue abril y mayo de 2015 (Figuras 3 y 5).

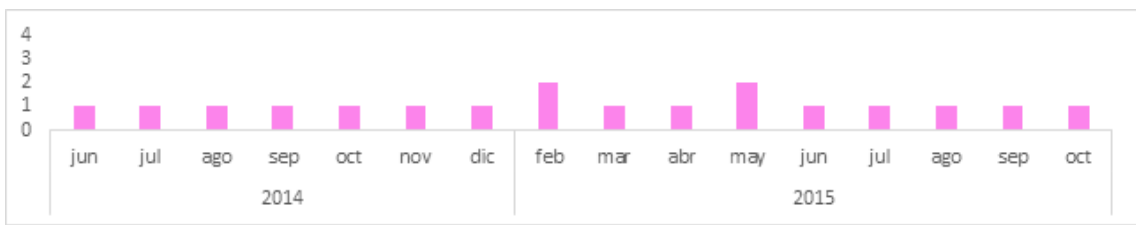

Individuo 1

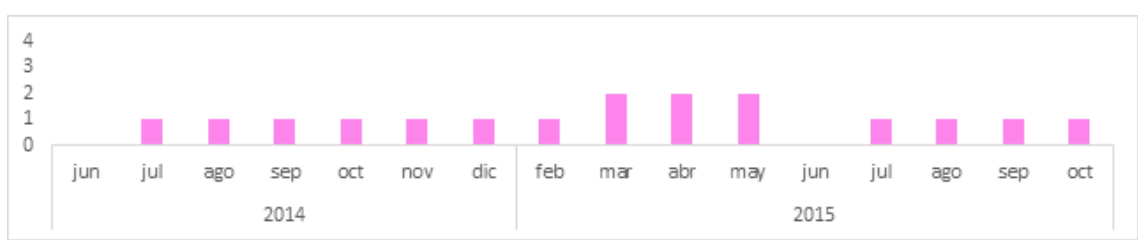

Individuo 3

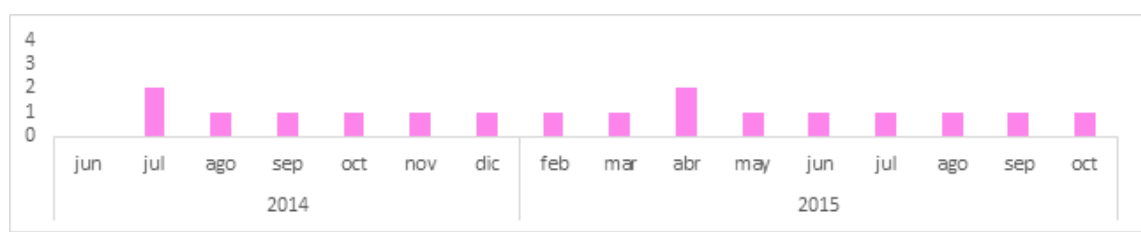

Individuo 5

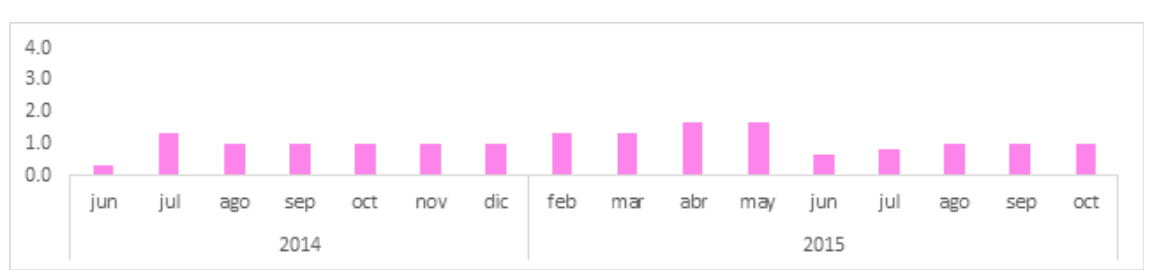

Promedio

Figura 5. Promedio total $(\mathrm{T})(\mathrm{n}=10)$ de brotes en tres de los 10 individuos estudiados $\mathrm{y}$ tomados al azar $(1,3,5$ de $W$. insignis). 
En promedio, $W$. insignis floreció en todo el tiempo investigado, sin embargo, en el individuo 1 la floración no estuvo presente en junio y julio 2015, en el espécimen 5 tampoco se manifestó en junio y julio 2014, ni en el cuarto, quinto y noveno mes de 2015. Los árboles 1 y 3 registraron en septiembre 2014 un pico máximo de floración de 4 y en el sujeto 3, se obtuvo ese mismo valor en los meses de septiembre y octubre (Figuras 3 y 6 ).

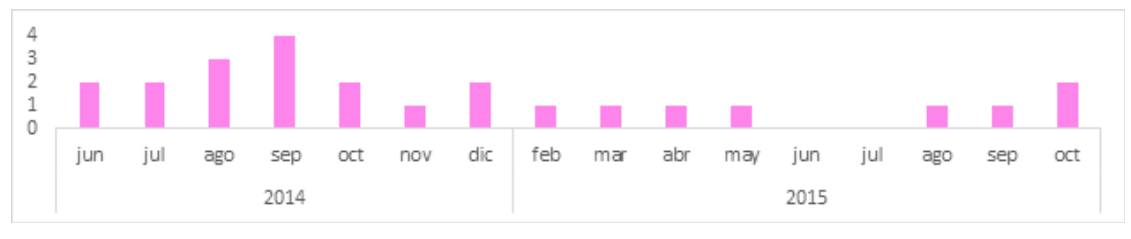

Individuo 1

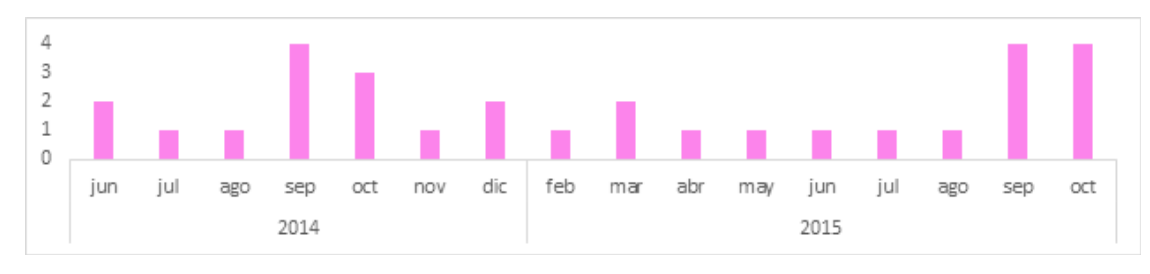

\section{Individuo 3}

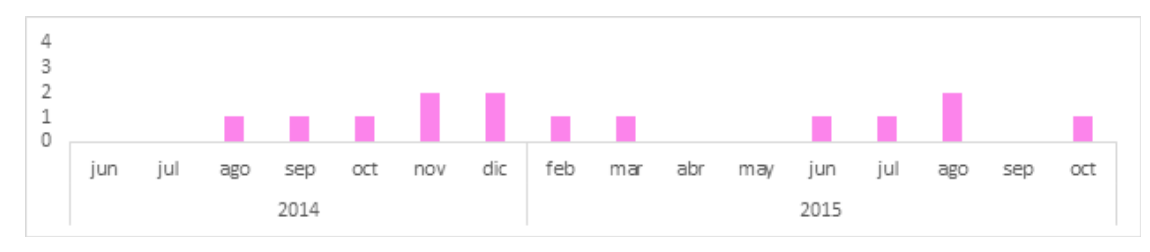

Individuo 5

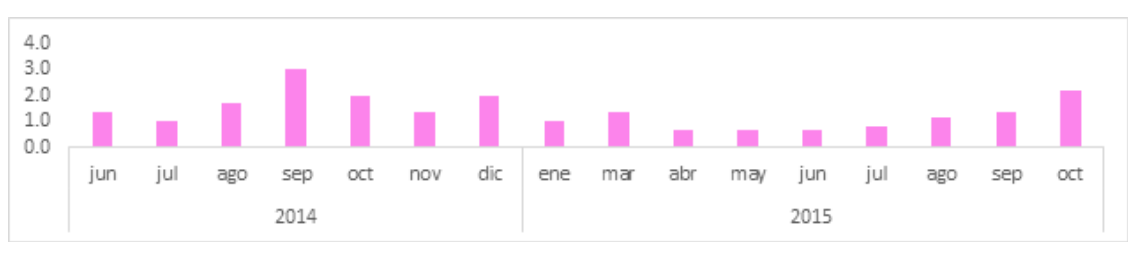

Promedio

Figura 6. Promedio total $(\mathrm{T})(\mathrm{n}=10)$ de flores en tres de los diez individuos estudiados y tomados al azar (1, 3, 5 de $W$. insignis). 
Esta especie presentó tres periodos de fructificación: de junio a septiembre (2014), de noviembre 2014 a mayo 2015 y de julio a octubre 2015. Los valores de la media fueron de o a 2.7, mientras en el individuo 1 y 3 fueron de o a 4 (Figura 3 y 7 ).
El individuo 5 presentó menos meses de fructificación ( $7 \mathrm{de}$ 16) que los especímenes 1 y 3 . Mientras tanto, el desarrollo de frutos en el árbol 1 estuvo presente durante 12 meses y en el árbol 3, durante 13 de ellos. Los ejes 1 y 3 mostraron mayor surgimiento de frutos en junio 2014 y octubre 2015 con un valor de 4 en esos lapsos (Figura 3 y 7 ).

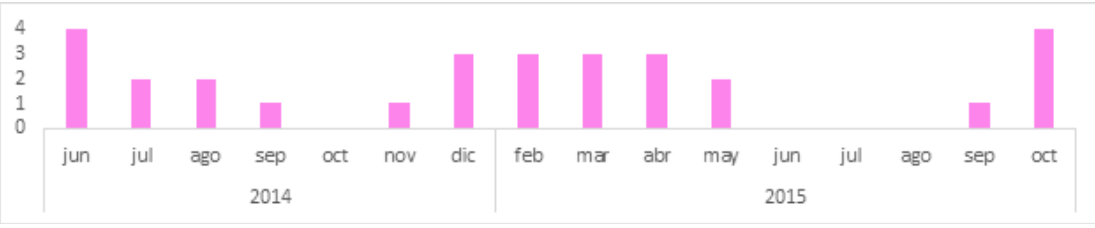

Individuo 1

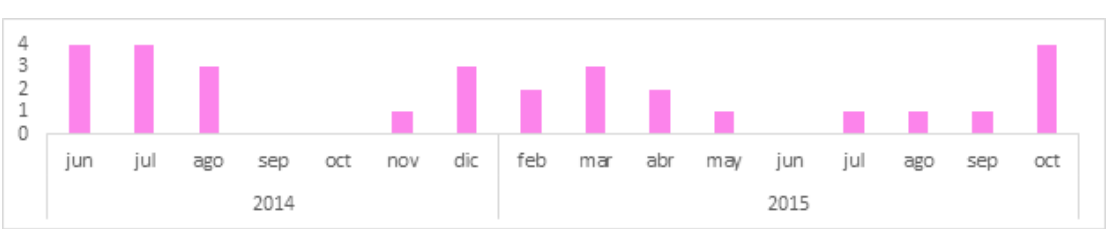

Individuo 3

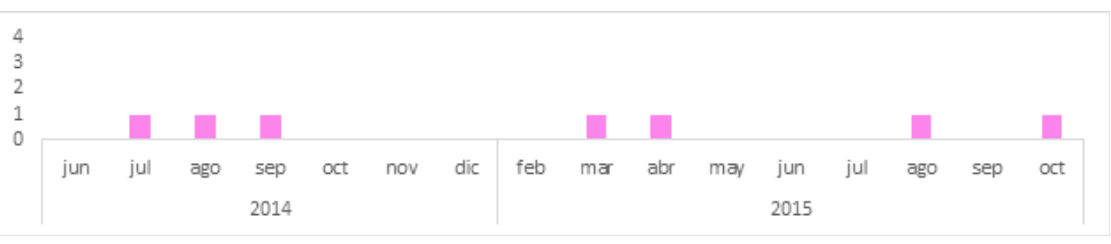

Individuo 5

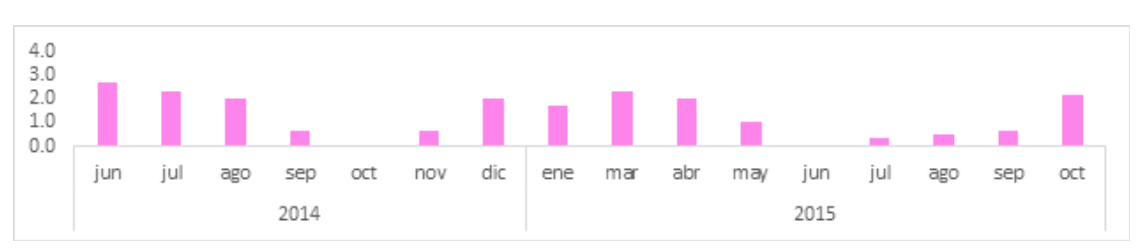

Promedio

Figura 7. Promedio total (T) $(\mathrm{n}=10)$ de tres de los 10 individuos estudiados y tomados al azar (1, 3, 5 de $W$. insignis). 
La caída de follaje de este árbol estuvo correlacionada con la precipitación ( $\mathrm{n}=10, \mathrm{r}=0.35, \mathrm{p}<0.05)$. Otro aspecto destacable, es que el surgimiento de flores también se asoció con las lluvias ( $\mathrm{n}=10, \mathrm{r}=0.46, \mathrm{p}<0.05)$ y en cambio, el nacimiento de frutos tuvo un vínculo inversamente proporcional con dicho fenómeno $(\mathrm{n}=10, \mathrm{r}=-0.51, \mathrm{p}<0.05)$ (Figura 8).

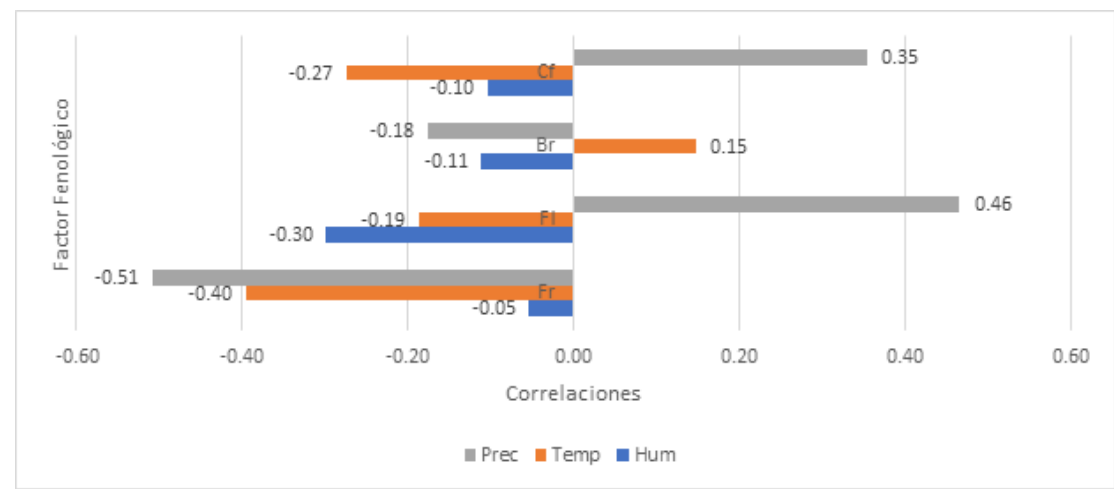

Figura 8. Correlaciones lineales ( $\mathrm{n}=10)$ entre 1: caída de follaje (cf), 2: brotes (br), 3: nacimiento de flores (fl), 4: surgir de frutos (fr) y precipitación (Prec), temperatura (Temp) y humedad relativa (Hum), en $W$. insignis, Reserva Biológica Alberto Manuel Brenes, Costa Rica.

\section{Discusión}

En el bosque de la ReBAMB existen diversas especies que presentan caída de follaje durante todo el año, como Warszewiczia uxpanapensis (Rubiaceae) (Brenes y Di Stefano, 2001) y Talauma gloriensis (Magnoliaceae) (Brenes y Sánchez, 2017), lo cual coincide con el comportamiento mostrado por $W$. insignis en esta investigación. La caída de follaje es una actividad constante en especies de árboles de Bosque Tropical Húmedo (Ortiz y Fournier, 1983). Lo anterior podría deberse a que los cambios en clima no son tan marcados como en otras zonas de vida, por ejemplo en el Bosque Tropical Seco, en el cual hay estaciones secas y lluviosas definidas y prolongadas.

Por otro lado, la floración de $W$. insignis es subanual de acuerdo con la clasificación de Newstrom, Frankie y Baker (1994), es decir, tiene más de un ciclo por año. Según Richards (1996) las especies tropicales "siempre en flor" son pocas, la mayoría lo hacen de manera intermitente, una o más veces al año o en intervalos más largos. Este individuo podría haber desarrollado una estrategia con polinizadores y tener disponible este recurso durante el año. De acuerdo con la lista de plantas elaborada por Fleming et al. (2009), W. insignis es polinizada por murciélagos, siempre presentes, los cuales requieren de la floración para alimentarse.

Esta característica de producir durante todo el año, sumada a que es un árbol con hasta $15 \mathrm{~m}$ de alto, de flores conspicuas, la hacen una especie atractiva como ornamento; aunque de acuerdo con Rojas, Bermúdez y Jiménez (2016) se ha empleado muy poco en este sentido a pesar de su alto potencial.

Al comparar W. insignis con otras especies de Malvaceae como Gossypium tometosum, planta endémica de Hawaii, se observó que también es subanual, porque su floración comienza en enero y febrero después de la temporada de lluvias, con picos en mayo, e inclusive continua en agosto y septiembre (Pleasants y Wendel, 2010); además de A. mandonii, cuya floración es todo el año (Tate, 2003). Contrario a $W$. insignis, otras muestras de Malvaceae encontradas en Los Andes (Bolivia, Chile y Perú) como 
Andeimalva spiciformes, florece de febrero a junio, $A$. machupichenccis, de diciembre a junio y $A$. chilensis de diciembre a febrero (Tate, 2003). Al parecer, muchas de las especies de Malvaceae muestran floración durante fases prolongadas del año.

La productividad de frutos de $W$. insignis evidencia periodos extensos, lo cual posiblemente acontece como respuesta para mejorar la dispersión de frutos y semillas, ya sea por la dehiscencia de sus frutos, la acción del viento (Ortiz y Fournier, 1983) o por el agua (Hidrocoria); permisible en este caso ya que los especímenes de la muestra se encontraban en el margen del río San Lorencito. Las especies de $W$. insignis tuvieron diferencias en su fructificación, concordante con García y Di Stefano (2004) en un estudio fenológico de Sideroxylon capiri (Sapotaceae) en un Bosque Seco Tropical de Costa Rica, en donde el surgimiento de frutos puede ocurrir todos los años y en cualquier época, con variabilidad tanto entre las estaciones como entre los individuos.

Tate (2003) refuerza lo anterior con un trabajo realizado en los Andes (Bolivia, Chile y Perú) en donde menciona que Andeimalva spiciformes fructifica febrero a junio, A. machupichenccis de diciembre a junio y A. mandonii (Malvaceae) todo el año, esta última muy parecida a $W$. insignis. Caso contrario, Eriotheca saxicola (Malvaceae) investigada por Carvalho-Sobrinho (2013) en el estado de Espirito Santo al este de Brasil, la cual evidencia fructificación más puntual en julio, septiembre y enero.

Al darse las cuatro fenoetapas en forma simultánea, dichos árboles invierten mucha energía en estos procesos, por lo que los valores no pueden alcanzar picos máximos. De esta manera, se observó una especie tanto con crecimiento vegetativo como con reproducción sexual continua.

Ahora bien, se presentan disimilitudes en el comportamiento fenológico de la muestra por diferencias genéticas. Los aspectos biofísicos como temperatura, humedad relativa, precipitación y suelo, son muy parecidos para todos los individuos estudiados.
En $W$. insignis se perciben asociaciones entre caída de follaje y el nacimiento de flores en relación con la precipitación (de julio a diciembre 2014 muy evidente y julio a octubre 2015 con patrones establecidos, Figura 3). El surgir de los frutos tuvo una correlación inversamente proporcional con la lluvia, lo que también fue hallado en Talauma gloriensis (Magnoliaceae) en el mismo tipo de bosque de la ReBAMB (Brenes y Sánchez 2017).

\section{Referencias}

Abdallah, M. A. et al (2011) A comparative study on red and white karade ( $H$. sabdariffa $L$.) calyces, extracts and their products. Pakistan Journal of Nutrition 10 (7), 680-693.

Boyle, A. y Bronstein, J. (2012). Phenology of tropical understory trees: patterns and correlates. Rev.biol.trop 6o (4), 1415-1430. http:// www.scielo.sa.cr/scielo.php?script $=\mathrm{sci}_{-}$ arttext\&pid=So034-77442012000400002

Brenes Cambronero, L. (1999). Autoecología de Elaeagia uxpanapensis D. Lorence (Rubiaceae), en la Reserva Biológica Alberto Manuel Brenes, San Ramón, Costa Rica. Tesis de Maestría. Universidad de Costa Rica, San Pedro de Montes de Oca, San José, Costa Rica. p.116.

Brenes Cambronero, L. y Di Stefano, J.F. (2001). Comportamiento fenológico del árbol Elaeagia uxpanapensis (Rubiaceae), en un Bosque Pluvial Premontano de Costa Rica. Rev.Biol.Trop. 49 (34), 989-999.

Brenes Cambronero, L. y Sánchez, R. (2017). Fenología de Talauma gloriensis Pittier (Magnoliaceae), Reserva Biológica Alberto Manuel Brenes, San Ramón, Alajuela, Costa Rica. Revista Pensamiento Actual 17 (1), 11-22.

Carvalho-Sobrinho, J. (2013). A new species of Eriotheca (Malvaceae: from Espirito Santo, Eastern Brasil. Phitotaxa. 108 (1), 49-53. 
Cheng, Z. (2001). Kenaf research products and applications in Japan. Plant fibres and products, 23(3), 16-24.

Estrada-Conteras, I., Equihua, M., Castillo-Campos, G. y Rojas-Soto, O. (2015) Climate Change and effects on vegetation in Veracruz, México: An approach using ecological niche modelling. Acta Botánica Mexicana. 112, 73-93.

Fleming, T., Geiselman, C. y Kress, W. (2009). The evolution of bat pollination: a phylogenetic perspective. Annals of Botany 104 (6), 10137-1043.

Fournier, L. (1974). Un método cuantitativo para la medición de características fenológicas en árboles. Turrialba, 24 (4), 422-423.

Fournier, L. y Charpantier, C. (1975). El tamaño de la muestra y la frecuencia de las observaciones en el estudio de las características fenológicas de los árboles tropicales. Turrialba 25 (1), 45-48.

Fryxell, P. (1981). Revision and expansion of the Neotropical Genus Wercklea (Malvaceae). Journal of the Arnold Arboretum 62 (4), 457-486.

García, E. y Di Stefano, J.F. (2004). Fenología de árbol Sideroxylon capiri (Sapotaceae) en el Bosque Seco Tropical de Costa Rica. Rev. Biol. Trop, 53 (1-2), 5-14.

Gómez, R. (2010). Fenología reproductiva de especies forestales nativas presentes en la jurisdicción de CORANTIOQUIA, un paso hacia su conservación. Medellín (Colombia): Volumen I, 2010, 177-180.

Huxley, P.A. (1983). En: P. A. Huxley. Phenology of tropical woody perennials and seasonal crop plants with reference to their management in agroforestry systems. Plant research and agroforestry. International Center for Research in Agroforestry. Nairobi (Kenya). 8-15, 503-525.
LaRotta, C. (1987). La etnobotánica: una aproximación metodológica desarrollada con la comunidad indígena Miraña (Amazonas Colombia). Memorias del primer simposio colombiano de etnobotánica. Corporación de Araracuara, Editorial Gente Nueva, Bogotá, Colombia.

Mahadevan, N. and Shivali, K.P. (2009). Hibiscus sabdariffa L. An overview, Natural Product Radiance, 8, 77-83

Mohta, D. (2000). Refiner Mechanical Pulping of Kenaf fibre: Such a process might reduce the demand for expensive softwood kraff pulps. Pulp and paper can. 101(8), 27-31.

Mooney, H.A., Bjorkman, A.E., Medina y Tomlinson, P.B. (1980). The study of physiological ecology of tropical plants -current status and needs. BioScience 30, 22-26.

Newstrom, L.E., Frankie, G.W. y Baker, H.G. (1994). "Una nueva clasificación para la fenología de plantas basada en patrones de floración en los árboles de tierras bajas del Bosque Lluvioso, de la Selva, Costa Rica”. Biotrópica 26 (2), 141- 159.

Odigie, J.P. y Adigun, S. (2003) Chronic administration of aqueous extract of Hibiscus sabdariffa attenuates hypertension rats. Journal of Ethnopharmacology $86(2-3), 181-185$.

Ortiz, R. y Fournier, L.A. (1983). Comportamiento fenológico de un Bosque Pluvial de Premontano en Cataratitas de San Ramón, Costa Rica. Rev. Biol. Trop. 31 (1), 69-74.

Pérez, C.A., Villalba, J.C. y Almanza, M.I. (2011). Fenología del roble (Quercus humboldtii bonpland). Biotecnología en el Sector Agropecuario y Agroindustrial, Edición Especial No. 2, Popayán, Cauca, Colombia. 145-154. 
Pérez, R., Condit, R. (2017). Tree Atlas of Panama. Smithsonian Tropical Research Institute. [Consulta 9 de octubre de 2020]. https:// stricollections.org/portal/taxa/index. php?taxon $=70915 \&$ clid $=71$

Pleasants, J. y Wendel, J. (2010). Reproductive and pollination biology of the endemic Hawaiian cotton, Gossypium tomentossum (Malvaceae). Pacific Science 64 (1), 45-55.

Richards, P. W. (1996). The Tropical Rain Forest. Cambridge, Londres.

Rojas, F., Bermúdez, G. y Jiménez, Q. (2016). Plantas ornamentales del trópico. (2da ed.) Editorial Tecnológica de Costa Rica, Cartago, Costa Rica.

Rondón, J. (1992). Hábito fenológico de 53 especies arbóreas del Jardín Botánico de San Juan de Lagunillas. Revista Forestal Venezolana 36, 2333 .

Sáenz, J., Nassar, M. (1965). Phytochemical Screening of Costa Rican Plants: Alkaloid Analysis II. Rev. Biol. Trop. 13 (2), 207-212.

Standley, P.C. (1937). Flora of Costa Rica, Part II. Studies of tropical American phanerogams. Botanical Series, 18(2), 491.

Standley, P.C., Pittier,H. (1916). Wercklea. Herb. 18,112.

Tate, J. (2003). Andeimalva, a new genus of Malvaceae from Andean South America. Lundella. 2003 (6), 10-18.

\section{Agradecimientos}

Se agradece a la Vicerrectoría de Investigación y Coordinación de Investigación de la Sede de Occidente, ambas de la Universidad de Costa Rica, por el financiamiento para realizar la presente investigación. También a Brayan Morera por su apoyo en las labores de campo, a Kevin Chavarría Esquivel por la confección del mapa, a Juan Gabriel Parra López por la ilustración y a Ronald Sánchez Brenes por su apoyo. 